DOI: $10.5455 / 2320-1770 . i j r \operatorname{cog} 20130632$

\title{
Recurrent vulval fibroepithelial polyp with pregnancy: a rare presentation
}

\author{
Meena Armo*, Sarita Agrawal, Manoj Minj, Kavita Babbar
}

Department of Obstetrics \& Gynecology, Govt. Chhattisgarh Institute of Medical Sciences (CIMS), Bilaspur, Chhattisgarh-495001, India

Received: 5 April 2013

Accepted: 14 April 2013

\section{*Correspondence:}

Dr. Meena Armo,

E-mail: drmeenaarmo@gmail.com,priya_d143@yahoo.com

(C) 2013 Armo M et al. This is an open-access article distributed under the terms of the Creative Commons Attribution License, which permits unrestricted use, distribution, and reproduction in any medium, provided the original work is properly cited.

\section{ABSTRACT}

A fibroepithelial polyp (FEP) is a relatively uncommon benign condition of vulva, mainly of reactive origin, occurs in women of reproductive age, mostly during pregnancy. Here is a case which presented with bilateral vulval mass, for that she underwent surgical excision. Histopathological examination revealed bilateral fibroepithelial vulval polyp. After 4 years of uneventful interval same female came with term pregnancy with a huge vulval mass on left side, having similar characteristic features as previous one. Emergency cesarean section followed by excision of polyp in the same sitting done. Histopathological report confirmed the finding of fibroepithelial polyp this time also. Only few cases of recurrent fibroepithelial polyp of vulva associated with pregnancy have been reported till date.

Keywords: Fibroepithelial polyp (FEP), Vulva, Pregnancy

\section{INTRODUCTION}

A fibroepithelial polyp (FEP), which is also referred to as skin tag or acrochordon, described originally by Norris and Taylor in $1966 .^{1}$ It is a relatively uncommon benign condition of vulva. It is mainly of reactive origin occurs in women of reproductive age, mostly during pregnancy. Majority are single and small in size, remain asymptomatic, however multiple or atypical forms are particularly associated with pregnancy, may produce cosmetic problems and can be misinterpreted as malignancy. ${ }^{2,3}$ We present a case of a bilateral vulval fibroepithelial polyp and recurred lesion with a brief review of literature.

\section{CASE REPORT}

A 24 year old married nulliparous woman presented on September 2008 with huge bilateral vulval mass for 3 years. Examination confirmed soft to firm, nontender, rubbery polypoidal mass measuring $15 \mathrm{~cm} \times 9 \mathrm{~cm} \times 5 \mathrm{~cm}$ involving whole of the right labia minora (Figure 1a). The left majora was also appearing as mass of $8 \mathrm{~cm} \times 5 \mathrm{~cm} \times 3 \mathrm{~cm}$ size with firm consistency. There was no other associated abnormalities or dermatoses detected. She had not given any history of hormonal intake. After thorough investigation, bilateral surgical excision of mass done under spinal anesthesia without significant blood loss. Histopathological examination revealed hypocellular stroma composed of spindle cells firmly set within a loose collagenous mixoid background with capillary proliferation thus confirmed benign fibroepithelial vulval polyp. Her postoperative period was uneventful. After 4 years of interval on April 2012 she came with complain of labour pain with vulval mass for 1 year. She had a history of delivery of male child by caesarian section 2 years back. Her previous pregnancy was uneventful (without such lesion). On examination she was found to have a huge vulval mass $16 \mathrm{~cm} \times 12 \mathrm{~cm} \times 10 \mathrm{~cm}$ in size involving lower half of the left labia majora (Figure 1b), having similar characteristic features as previous one. Emergency Cesarean section followed by excision of the polyp done in the same sitting under spinal anesthesia. She was discharged after 
removal of stitches on $10^{\text {th }}$ day. Histopathological report confirmed the finding of fibroepithelial polyp this time also.

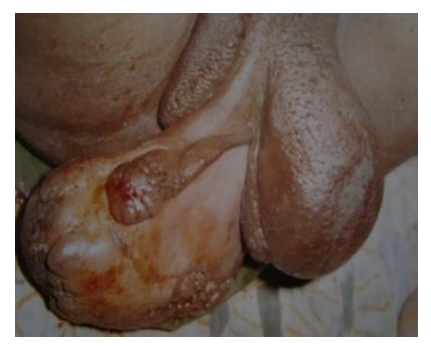

(a)

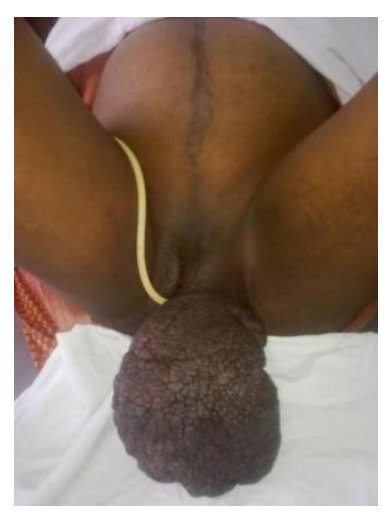

(b)

Figure 1: Fibroepithelial vulval polyps.

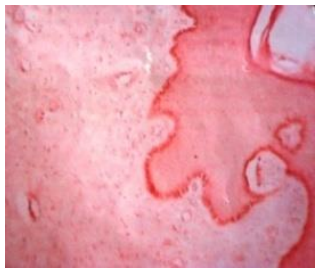

(a)

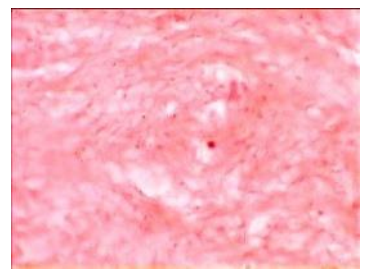

(b)
Figure 2: Hypocellular stroma composed of spindle cells firmly set within a loose collagenous mixoid background with capillary proliferation.

\section{DISCUSSION}

The fibroepithelial stromal polyp was described originally by Norris and Taylor in 1966 like a benign injury. ${ }^{1}$ It arises from proliferation of mesenchymal cells within the hormonally sensitive subepithelial stromal layer of the lower female genital tract of mostly reproductive age group. ${ }^{3}$ Apart from the pregnancy it has also been reported in postmenopausal females following $\mathrm{HRT}^{2}$ and females having dermatosis. ${ }^{5}$ The clinical presentation may vary from small flesh colored, hypo or hyperpigmented sessile growth to large pedunculated polypoid formation or finger like projections, it is more frequent in the vagina followed of vulva and cervix. The size varies between $1-2 \mathrm{~cm}$ to $15-$ $20 \mathrm{~cm} .{ }^{2,4}$ Histologically the most characteristic feature of
FEP is presence of multinucleated stromal cells which are most commonly seen near the epithelial-stromal interface or adjacent to the prominent central vasculature. FEPs may be of two types: one that is predominantly epithelial and another is primarily stromal, the stromal cellularity of polyp can be variable. The hypocellular variant is composed of spindle cells set with in a loose collagenous myxoid like stroma. The hypercellular form exhibits nuclear pleomorphism and mitotic activity, thereby mimicking a malignant process. ${ }^{4}$ The pathogenesis has not been clarified yet; however evidences suggest that influx hormone plays a role, therefore small FEP associated with pregnancy regress spontaneously after delivery. ${ }^{5}$ Immunohistochemically FEPs are often positive for desmin, vimentin, estrogen, and progesterone receptors and less frequent for actin. ${ }^{2}$ The treatment of choice is surgical excision. Local recurrence may occur if incompletely excised or if there is continuous hormonal stimulation (e.g. pregnancy, tamoxifen). ${ }^{2,6}$ Thus wide range of morphological appearance of FEP needs expert pathological interpretation to exclude other site specific lesions such as deep aggressive angiomyxoma, angiomyofibroblastoma, cellular angiofibroma, sarcomas. 2,4

\section{CONCLUSION}

FEPs of vulva have been reported by various authors previously, however the largest size reported was $28 \times 27 \times 12 \mathrm{~cm}$ in a 16 year old women. ${ }^{7}$ In our case, size of polyp which recurred in pregnancy was $16 \times 12 \times 10 \mathrm{~cm}$ and of hypocellular variant without atypia. Our case is a unique as FEPs are rarely bilateral ${ }^{8}$ and only few cases of recurrent fibroepithelial polyp of vulva associated with pregnancy have been reported till date.

Consent to publish these images has been obtained from the patient.

Funding: No funding sources

Competing interests: None declared

Ethical approval: Not required

\section{REFERENCES}

1. Norris HJ, Taylor HB. Polyps of the vagina. The benign injury resembling sarcoma botryoides. Cancer 1966;19:227-32.

2. Nucci MR, Young RH, Fletcher CD. Cellular pseudosarcomatous fibroepithelial stromal polyps of the lower female genital tract: An under recognized injury often misdiagnosed as sarcoma. Am J Surg Pathol 2000;24:231-40.

3. Halvorsen TB, Johannesen E. Fibroepithelial polyps of the vagina: plows they old granulation tissue polyps? J Clin Pathol 1992;45:235-40.

4. Nucci MR, Fletcher CD. Vulvovaginal soft tissue tumors; updates and review. Histopathology 2000;36:97-108.

5. Dane C, Dane B, Cetin A, Erginbas M, Total Z. Association of psoriasis and vulval epithelial polyp. Am J Clin Dermatol 2008;9:333-5. 
6. Pearl Crombleholme WR, Green JR, Bottles K. Fibroepithelial polyps of the vagina in pregnancy, Am J Perinatol 1991;8:236-8.

7. Min Jung Oh, Min Jee Kee, Woo Dae Kang, Seok Mo Kim, Ho Sun Choi. A case of giant fibroepithelial polyp of vulva. Korean J Obstet Gynecol 2010;53:194-7.

8. Carter J, Elliott P, Russell P. Bilateral fibroepithelial polypi of labium minus with atypical stromal cells. Pathology 1992:37-9.

DOI: $10.5455 / 2320-1770$. ijrcog20130632

Cite this article as: Armo M, Agrawal S, Minj M, Babbar K. Recurrent vulval fibroepithelial polyp with pregnancy: a rare presentation. Int J Reprod Contracept Obstet Gynecol 2013;2:245-7. 